\title{
REPERCUSSÕES DO TESTE DE ENDURANCE RESPIRÁTORIO DETECTADAS POR ELETROMIOGRAFIA DE SUPERFÍCIE (EMGs) EM PACIENTES CANDIDATOS AO TRANSPLANTE HEPÁTICO
}

\author{
Effects of respiratory endurance test detected by surface electromyography (sEMG) \\ in patients candidates for liver transplantation
}

\begin{abstract}
Sulaine Rodrigues Custódio', Rafaela Brunetti dos Santos ${ }^{1}$, Áurea Maria Oliveira da Silva², Lígia dos Santos Rocetto Ratti', Ilka de Fátima Santana Ferreira Boin ${ }^{3}$, Rayssa Pistilli Duarte', Rodrigo Marques Tonella ${ }^{1}$
\end{abstract}

\section{RESUMO}

Objetivo: Analisar as repercussões que o teste de endurance da musculatura inspiratória com dispositivo Powerbreathe ${ }^{\circledR}$ repercute na função do diafragma através de valores de PImáx e de RMS (Root Mean Square) de eletromiográfica de superfície (EMGs) em pacientes no pré-operatório de transplante hepático. Materiais e Métodos: Estudo prospectivo realizado no Ambulatório do Gastrocentro do Hospital de Clínicas da UNICAMP. Foram selecionados 11 indivíduos candidatos a transplante hepático de ambos os gêneros, que assinaram o Termo de Consentimento Livre e Esclarecido (TCLE). Foram submetidos à avaliação antropométrica, prova de função pulmonar, medidas de PImáx e realização de eletromiografia de superfície. Resultados: Os valores de Pimáx aferidos mostraram que há diminuição da força muscular inspiratória tanto para homens quanto para mulheres. Na cúpula direita observouse que quando comparado o momento sem carga com o Tempo 1, houve redução significativa dos valores de RMS ( $p=0,008)$, e na comparação entre Tempo 1 e Tempo 2 houve aumento significativo do RMS $(p=0,006)$. Ao comparar-se o valor no momento sem carga com o Tempo 1 na cúpula esquerda, houve redução significativa dos valores de RMS ( $p=0,0009$ ), e na comparação entre Tempo 1 e Tempo 2 , houve aumento significativo do RMS ( $p=$ 0,0009). Conclusão: Foi possível observar que os valores de PImáx não foram capazes de detectar a fadiga imposta pelo teste. Em contrapartida, os valores de EMS mostraram-se sensíveis em detectar a fadiga da musculatura diafragmática pós-teste de endurance.

Descritores: Transplante Hepático, Eletromiografia, Exercícios Respiratórios, Fisioterapia.

Instituições:

${ }^{1}$ Unidade de Terapia Intensiva de Transplante Hepático do Hospital de Clínicas - Universidade Estadual de Campinas - Campinas/SP

2 Unidade de Transplante Hepático Hospital de Clínicas -

Universidade Estadual de Campinas; Campinas/SP

${ }^{3}$ Cirurgia do Fígado e Transplante de Fígado da FCM / UNICAMP, Campinas/SP

\section{Correspondência}

Rayssa Pistilli Duarte

Rua José Miguel Saker Filho, 83 - Apto.132 - CEP 18010-090 -

Sorocaba/SP - Brasil

Tel.: (15) 32275986 / (15) 99121-9485.

E-mail: rayssa.pistilli@gmail.com

Recebido em: 13/12/2016
Aceito em: 13/01/2017

\section{INTRODUÇÃO}

O fígado é um órgão vital que faz parte do sistema digestório, tendo como principal função a digestão, além da metabolização e armazenamento de nutrientes; seu funcionamento inadequado pode gerar inúmeras complicações, sendo a mais comum a insuficiência hepática, doença que pode se instalar de forma crônica ou aguda, ambas caracterizadas pela incapacidade do fígado em exercer suas funções. ${ }^{1,2}$

Existem inúmeras condutas para as diferentes doenças hepáticas, sendo elas clínicas, ou cirúrgicas; dentre as condutas cirúrgicas, pode-se destacar o transplante 
hepático como a que apresenta melhor evolução para pacientes seriamente comprometidos com doença em fase terminal. Após avaliação multidisciplinar e acompanhamento, o paciente é listado na fila de transplante de acordo com tipo sanguíneo, idade, peso e gravidade da disfunção hepática, medida através da escala de MELD (Model for End-Stage Liver Disease). Quanto maior a pontuação, maior é a prioridade de alocação do paciente para a realização da substituição do órgão acometido. ${ }^{2,3}$

Quanto maior o comprometimento da função hepática, maior é a propensão à fadiga respiratória, considerada uma das maiores complicações do pós-operatório. Diante disso, fazem-se necessárias intervenções antes e após a abordagem cirúrgica, com proposta terapêutica de treinamentos da musculatura respiratória minimizando complicações. ${ }^{4,5}$

A eletromiografia de superfície (EMGs) é um método diagnóstico não invasivo, em que sinais dos picos gráficos da atividade muscular voluntária são captados através de eletrodos conectados na superfície da pele. Sua utilização pode ser realizada durante o teste de endurance muscular respiratório. ${ }^{6}$

O endurance muscular respiratório pode ser medido através de teste de esforço contra uma resistência muscular inspiratória imposta pelo equipamento threshold, com carga calculada em $30 \%$ da Pressão Inspiratória Máxima (PImáx), durante dois minutos. Atualmente, o treinamento muscular inspiratório apresentou avanço tecnológico importante e novos instrumentos estão disponíveis para utilização. ${ }^{7-9}$

O dispositivo eletrônico Powerbreathe ${ }^{\circledR}$ é indicado para o treinamento da musculatura inspiratória através de alterações do fluxo em diferentes intensidades de cargas ajustadas através da PImáx. Além do treino de força muscular, existe também a possibilidade de treinamento do endurance da musculatura respiratória; para que haja maior especificidade do treinamento, faz-se necessário o teste de endurance da musculatura respiratória, com intuito de detectar possíveis disfunções na capacidade contrátil muscular contra uma carga ajustada em determinado tempo. ${ }^{7,9-11}$

\section{MATERIAIS E MÉTODOS}

Estudo prospectivo, realizado no Ambulatório do Gastrocentro do Hospital de Clínicas da UNICAMP durante o segundo semestre de 2016, com aprovação prévia do comitê de ética da Universidade Estadual de Campinas, com parecer número: 922/2009. Os pacientes que concordaram em participar da pesquisa leram e assinaram previamente o Termo de Consentimento Livre e Esclarecido (TCLE). Foram selecionados 11 indivíduos candidatos a transplante hepático em acompanhamento no ambulatório de ambos os gêneros, com idade $\geq 18$ anos, com escore MELD superior a 10 e conscientes, orientados, após realização de provas de funções pulmonares com sucesso, seguindo-se a realização do teste de endurance. Foram excluídos pacientes incapazes de realizar as provas de funções pulmonares, com MELD <10, não listados na fila de transplante hepático.

Os pacientes foram submetidos à avaliação antropométrica, prova de função pulmonar e medidas de PImáx. Para a realização da eletromiografia de superfície, o paciente permaneceu em decúbito dorsal e foram posicionados quatro eletrodos auto-adesivos da marca $3 \mathrm{M}^{\circledR}$ do Brasil, sendo dois na região paraxifóide a $5 \mathrm{~cm}$ do processo xifóide e dois na região da margem costal bilateral.

A aquisição dos sinais foi feita em uma frequência de $300 \mathrm{~Hz}$ e a sensibilidade de amplitude do sinal de 300 $\mu \mathrm{V}$ antes, durante e ao final do teste de endurance, que teve duração total de $2 \mathrm{~min}$, com intervalo de 30 segundos para descanso do paciente, padronizados a seguir:

Sem carga - durante os primeiros dez segundos de inspirações e expirações profundas do paciente, sem carga, guiadas por comandos verbais padronizados, sendo analisado o maior valor atingido durante o período;

Tempo 1 - a segunda medida foi repetida durante os primeiros dez segundos do teste de endurance com dispositivo eletrônico Powerbreathe ${ }^{\circledR}$ ajustado com carga de $30 \%$ da PImáx, num total de um minuto;

Tempo 2 - o terceiro sinal, após 30 segundos, de intervalo foi colhido nos últimos dez segundos do teste de endurance, que teve duração total de dois minutos.

\section{RESULTADO}

A amostra foi composta de 13 indivíduos, dos quais dois foram excluídos por não conseguirem concluir 0 teste, participando do estudo 11 pacientes, sendo $82 \%$ do sexo masculino (nove pacientes) e dois pacientes do sexo feminino. A média das idades foi de $58 \pm 7,4$ anos e todos os participantes realizaram previamente prova de função pulmonar. As características dessa amostra estão descritas numericamente na tabela 1.

Quando observada a alteração na SpO2 durante o teste de endurance, verificou-se que ocorreu aumento desses valores com $p=0,008$, quando comparado com o inicial, antes da realização dos exercícios e com o final, após a realização do exercício, porém sem relevância clínica. 
Sulaine Rodrigues Custódio, Rafaela Brunetti dos Santos, Áurea Maria Oliveira da Silva, Lígia dos Santos Rocetto Ratti, Ilka de Fátima Santana Ferreira Boin, Rayssa Pistilli Duarte1, Rodrigo Marques Tonella

Tabela 1: Características da descritiva da amostra estudada

\begin{tabular}{lrrrc}
\hline Característica & Mínimo & Máximo & Média & $\begin{array}{l}\text { Desvio } \\
\text { Padrão }\end{array}$ \\
\hline Altura (m) & 160.00 & 184.00 & 1.72 & 7.97 \\
Peso (kg) & 66.00 & 98.00 & 76.09 & 9.94 \\
Idade (anos) & 41.00 & 68.00 & 57.00 & 7.50 \\
IMC (kg/m2) & 21.31 & 31.21 & 25.82 & 2.67 \\
FVC (I/min) & 35.00 & 90.00 & 77.00 & 14.79 \\
VEF1 (I/min) & 34.00 & 95.00 & 83.00 & 16.81 \\
PF (I/s) & 29.00 & 194.00 & 129.00 & 50.22 \\
\hline
\end{tabular}

IMC = Índice de Massa Corporal; FCV = Capaciade Vital forçada;

$V E F 1=$ Volume Expiatório Final; PF = Pico de fluxo

Foi avaliada a alteração da frequência cardíaca durante o exercício e verificou-se que ocorreu aumento dos valores de batimentos cardíacos, sendo que $75 \mathrm{bpm}$ (antes do exercício) subiram para 81 bpm após o mesmo $(p=0,0327)$.

Com relação aos valores de Plmáx aferidos, em comparação aos valores de normalidade para sexo e idade (Neder, 1999) há diminuição da força muscular inspiratória nos pacientes inclusos na amostra, tanto para os homens quanto para as mulheres, de acordo com a tabela 2 .

Tabela 2: Valores de Plmax aferidos e preditos de acordo com sexo e idade

\begin{tabular}{cccccc}
\hline $\begin{array}{c}\text { Homens } \\
\text { Idade (anos) }\end{array}$ & $\begin{array}{c}\text { PImax } \\
\text { Obtida }\end{array}$ & $\begin{array}{c}\text { PImax } \\
\text { Predita }\end{array}$ & $\begin{array}{c}\text { Mulheres } \\
\text { idade (anos) }\end{array}$ & $\begin{array}{c}\text { Plmax } \\
\text { Obtida }\end{array}$ & $\begin{array}{c}\text { Plmax } \\
\text { Predita }\end{array}$ \\
\hline 59 & -8 & -108 & 41 & -52 & -90 \\
54 & -37 & -112 & 65 & -52 & -78 \\
51 & -71 & -114 & & & \\
41 & -18 & -122 & & & \\
58 & -27 & -108 & & & \\
60 & -28 & -107 & & & \\
65 & -69 & -103 & & & \\
68 & -107 & -100 & & & \\
63 & -95 & -104 & & & \\
\hline
\end{tabular}

Plmax = Pressão inspiratória máxima

A comparação dos valores de Pimáx, antes e após o exercício demonstrou que em média não ocorreu alteração, pois antes do exercício, a média foi de 51,27 e após $51,09(p=0,721)$, valores expressos na tabela 3 .

Tabela 3: Alteração nos valores de Plmax durante o teste de endurance muscular

\begin{tabular}{|l|c|c|c|c|}
\hline \multicolumn{1}{|c|}{ Variável } & Mínimo & Máximo & Média & Desvio Padrão \\
\hline Plmax Inicial & 8.00 & 107.00 & 51.27 & 31.68 \\
\hline Plmax Final & 10.00 & 98.00 & 51.09 & 30.31 \\
\hline
\end{tabular}

Plmax = Pressão inspiratória máxima
A alteração dos valores do RMS da cúpula diafragmática direita foi representada na figura 1 nos três momentos de aferição. Verificou-se que, quando comparados os tempos sem carga e Tempo 2, esses momentos não apresentaram valores de comparação significativos com $p=0,0528$. No entanto, foi possível observar aumento numérico dos valores. Ao compararmos os valores de RMS dos momentos sem carga com o Tempo 1, foi possível evidenciar uma redução significativa dos valores de RMS ( $p=0,008)$, demonstrando uma melhora rápida do endurance muscular, que porém não se sustenta por muito tempo, já que na comparação seguinte dos valores de RMS entre Tempo 1 e Tempo 2 houve aumento significativo do RMS com $p=0,006$, mostrando uma piora importante da função muscular sob efeito da carga de treinamento de endurance.

Figura 1: Alteração do RMS da cúpula diagragmática direita durante o teste de endurance muscular nos três momentos de aferição.

\section{Eletromiografo na cúpula diafragmatica Direita Endurance $(\mu \mathrm{V})$}

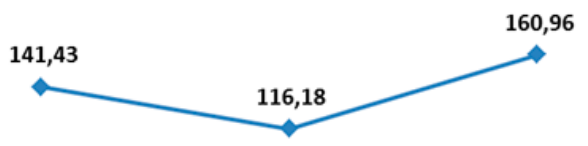

$\mathrm{Na}$ figura 2 foram representadas as alterações nos valores de RMS da cúpula diafragmática esquerda obtidos durante os três momentos de aferição. Quando comparados os momentos de aferição dos valores de RMS sem carga e Tempo 2, foi possível evidenciar valores não significativamente diferentes $(p=0,477)$; porém, foi observado o mesmo aumento numérico visto na cúpula direita. Ao se comparar os valores sem carga com o Tempo 1 na mesma cúpula, houve redução significativa no valor do RMS encontrado ( $p$ $=0,0009$ ), demonstrando melhora da função muscular diagramática, que também foi encontrada na cúpula diafragmática direita. Quando comparados os RMS do Tempo $1 \mathrm{com}$ o Tempo 2, houve a mesma piora da função muscular $(p=0,0009)$ sob efeito de carga no treinamento muscular, com aumento significativo do RMS.

Figura 2: Alteração do RMS da cúpula diagragmática esquerda durante o teste de endurance muscular nos três momentos de aferição.

Eletromiografo na cúpula diafragmatica Esquerda Endurance $(\mu \mathrm{V})$

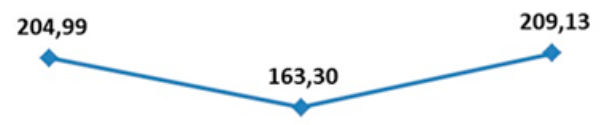


Ao se comparar as cúpulas diafragmáticas direita e esquerda, observou-se que não houve diferenças significativas entre os momentos sem Carga $(p=0,250)$, Tempo $1(p=0,250)$ e Tempo $2(p=0,309)$.

\section{DISCUSSÃO}

Dos artigos selecionados, oito estudavam a população iLimongi, ${ }^{12}$ em estudo com pacientes pré-transplante hepático, verificou predomínio do sexo masculino $(78,6 \%)$, o que corrobora com o presente estudo, em que a amostra apresentou prevalência de $82 \%$ do gênero masculino.

Os valores de espirometria não demonstraram a presença de doenças obstrutivas e/ou restritivas que pudessem interferir nos resultados do teste de endurance aplicado no protocolo. Em trabalho realizado por Garcia et. Al 11 no pré-transplante hepático, os valores médios de CVF e VEF1 apresentaram-se dentro da normalidade, ${ }^{11}$ observações que corroboram com o presente estudo.

Barcellos et al ${ }^{13}$ em 2008, notaram que candidatos ao transplante hepático não apresentaram redução da função pulmonar, mas evidenciaram perda da força muscular respiratória, através da medida de PImáx. Limong ${ }^{12}$ em seu estudo de 2014, analisou que pacientes hapatopatas apresentaram valores inferiores de PImáx ao predito, o que condiz com o presente estudo, que demonstrou valores de PImáx inferiores, comparados com os valores de normalidade,,$^{12,13}$ de acordo com sexo e idade, segundo Nedder et al. ${ }^{14}$

No presente estudo, não foram analisados pacientes sob desmame de ventilação mecânica, assim como no trabalho Chang et. $\mathrm{Al}^{8}{ }^{8}$ e sim candidatos ao transplante hepático extubados. Dessa forma, pode-se justificar o fato de não ter ocorrido redução da PImáx para pacientes deste estudo, porém, foi seguido o mesmo protocolo de avaliação do endurance muscular inspiratório correlacionando com o índice de resistência à fadiga.

Chang et al ${ }^{8}$ mensurou que após o teste de endurance houve diminuição da PImáx, estando relacionado ao índice de fadiga muscular respiratória. No presente estudo, não houve diferenças significativas dos valores ao se comparar antes e após o teste de endurance, justificando que não há perda da PImáx. ${ }^{9}$

Bisset et $\mathrm{Al}^{7}$ em 2015, aplicaram o teste de endurance muscular respiratório em pacientes após sete dias de ventilação mecânica, e $48 \mathrm{~h}$ de sucesso na extubação, verificando perda do endurance muscular, sem referência à sensação de dispneia, avaliados através da aplicação da escala de Borg Modificada contrapondo o presente trabalho, onde não houve perda do endurance através da verificação da PImáx.

Chang et. $\mathrm{Al}^{8} \mathrm{e}$ Bisset et. $\mathrm{Al}^{7}$ utilizaram o mesmo protocolo para realização de teste de endurance, sendo imposta resistência inspiratória equivalente à 30\% da PImáx inicial por um período de dois minutos, sem intervalos. No presente estudo, foi necessário fracionar o tempo com intervalo de 30 segundos entre o primeiro e o último minutos, devido ao relato de dispneia e a incapacidade de prosseguir.

As alterações frente ao teste de endurance foram avaliadas através da frequência cardíaca (FC) e saturação de oxigênio (SatO2), que apresentaram aumento significativo, porém sem relevância clínica, corroborando o estudo de Silva ${ }^{15} \mathrm{em}$ que houve aumento da SatO2 dentro dos valores de normalidade, portanto sem impacto ao paciente. ${ }^{13}$

Ao comparar a elevação de RMS no teste de endurance respiratório durante os três momentos, sendo eles Sem Carga, Tempo 1 e Tempo 2 em ambas as cúpulas, não houve aumento significativo, mas pode-se considerar maior diferença numérica entre o Tempo 1 e Tempo 2 na cúpula direita. Esse fato corrobora com o estudo de Silva (2014), 15 pois $53,2 \%$ de sua amostra apresentaram aumento do RMS da cúpula diafragmática direita, indicando fraqueza muscular.

Limong ${ }^{12}$ refere em sua pesquisa, que devido à perda de massa e força muscular respiratória apresentada por hepatopatas, faz-se necessário maior desempenho de força para vencer a resistência imposta, justificando a elevação da atividade elétrica do RMS obtida no presente estudo, no momento de aferição do tempo 2 , onde a musculatura foi posta sobre a carga de exercício muscular. $^{13}$

Verificou-se que o esforço máximo imposto pelo teste de endurance ultrapassa o nível de condicionamento da musculatura diafragmática do pré-transplante, já sarcopênica. Dessa forma, sugere-se a implementação de condicionamento muscular na frequência, força e tempo intermediários para melhorar a sarcopenia.

\section{CONCLUSÃO}

Observou-se que os valores de PImáx não determinaram valores compatíveis com perda de endurance muscular. Em contrapartida, os valores de RMS mostraramse sensíveis em detectar a perda de endurance da musculatura diafragmática pós-teste. 
Sulaine Rodrigues Custódio, Rafaela Brunetti dos Santos, Áurea Maria Oliveira da Silva, Lígia dos Santos Rocetto Ratti, Ilka de Fátima Santana Ferreira Boin, Rayssa Pistilli Duarte1, Rodrigo Marques Tonella

\section{ABSTRACT}

Purpose: To analyze the repercussions of the endurance test in the inspiratory muscle with Powerbreathe $₫$ device on the diaphragm through the IPmax and RMS (Root Mean Square) values of the surface electromyography (sEMG) in patients preoperatively to liver transplantation. Materials and Methods: Prospective study performed at the Gastrocentro Ambulatory of Clinical Hospital of UNICAMP. Eleven individuals candidates for liver transplantation of both genders, who signed the consent term were selected. They were submitted to anthropometric assessment, pulmonary function test, IPmax measurements and a surface electromyography performed. Results: The measured IPmax values showed that there is a decrease in inspiratory muscle strength both for men and women. In the right dome, it was observed that when the unloaded moment was compared to Time 1, there was a significant reduction in the RMS values $(p=0.008)$ and by comparing Time 1 and Time 2 , there was a significant increase in the RMS ( $p=$ 0.006). When comparing the value at the unloaded time with Time 1 in the left dome, there was a significant reduction in the RMS values $(p=0.0009)$, and in the comparison between Time 1 and Time 2, there was a significant increase in the RMS ( $p=0.0009)$. Conclusion: It was possible to observe that the IPmax values were unable to detect the fatigue imposed by the test. On the other hand, EMS values were sensitive in detecting diaphragmatic muscle fatigue after the endurance test.

Keywords: Thyroid, Hepatitis, Thyrotoxicosis, Liver, Inflammation.

\section{REFERÊNCIAS}

1. Teodoro V, Bragagnolo MJ, Lucchesi L, Condo M, Tufik S. Avaliação dos potenciais evocados relacionados a eventos (ERP-P300) em pacientes com cirrose hepática sem encefalopatia. Rev Gastroenterol. 2008:45(1): 82-86.

2. Mies S. Transplante de fígado. Rev Ass Med Brasil. 1998:44(2): 127-34.

3. Manual do paciente candidato ao transplante de fígado. Hosp Meridional. 2007.

4. Nitrini AMS, Stirbulov R, Rolim EG. Influência da ascite na avaliação da função pulmonar em portadores de hipertensão portal. J Bras Pneumol. 2004:30(1):22-27.

5. Guedes CAV. Avaliação do desempenho dos músculos respiratórios da capacidade funcional, da fadiga e da qualidade de vida em candidatos à transplante de fígado. [Tese em Fisiopatologia Cirúrgica]. Universidade Estadual de Campinas, 2011.

6. Silva AMO, Cliquet A, Boin IFSF. Profile of Respiratory Evaluation Through Surface Electromyography, Manovacuometry, and Espirometry in Candidates on the Liver Transplant Waiting List. J Transplantation Proceedings. 2012. 44: 2403-5

7. Bissett B, Leditschke IA, Boots R, Paratz J. Meaned but weary: One third of adult intensive care ptients machanically ventilated for 7 days or more have impaired inspiratory muscle endurance after successful weaning. J Heart \& Lung. 2015:44:15-20.

8. Chang AT, Boots RJ, Brown MG, Paratz J, Hodge PW. Reduced inspiratory muscle endurance following sucessful weaning from prolonged mechanical ventilation. J Chest. 2005(128):553-9.
9. Beckerman M, Magadle R, Weinr M, Weinr P. The effects of 1 year of specific inspiratory muscle training in patients with COPD. J Chest. 2005(128):3177-82.

10. Carvalho EM,Isern MRM, Lima PA, Machado CS, Biagini AP, Massarollo PCB. Força muscular e mortalidade na lista de espera de transplante de fígado. Rev Bras Fisioter São Carlos. 2008.12(3):235-40.

11. Garcia E, Silvério AO, Brandão AB, Moreira JS. Função pulmonar na cirrose hepática. J Pneumol. 1997; 23:225-30.

12. Limongi V. Estudo do perfil e dos efeitos de um programa de intervenção fisioterapêutica respiratória em candidatos á transplante de fígado. Tese de Mestrado apresentada à Faculdade de Ciências Médicas da Universidade Estadual de Campinas. 2014.

13. Barcelos S, Dias AS, Forgiarini Jr. LA, Monteiro MB. Transplante hepático: repercussões na capacidade pulmonar, condição funcional e qualidade de vida. Rev. Gastroenterol. 2008;45(3):186-91.

14. Neder JA, Andreoni S, Castelo-Filho A, Nery LE. Reference values for lung function tests. I. Static volumes. Braz J Med Biol Res. 1999;32(6):703-17.

15. Silva AMO. Eletromiografia de superfície de músculos respiratórios: Avaliação de hepatopatas em lista de espera de transplante de fígado e de não hepatopatas. Tese de doutorado apresentada à Faculdade de Ciências Medicas da Universidade Estadual de Campinas. 2014. 